\title{
Editorial: Volume 44 (issue 2), 2015
}

We are delighted to share with you the latest issue of Comparative and International Education. As in previous issues, we have another set of excellent articles on various comparative and international educational themes and topics, including once again an article in French. In reading over the articles, a common theme comes through - alternatives, which is refreshing in this day and age of standardization and homogenization of educational practices and approaches.

To begin with, we have two articles focusing on Canadian educational contexts, although the second one engages with educational discourses in China, as well as Canada. In the first article, "Exploring the Concepts of Traditional Inuit Leadership and Effective School Leadership in Nunavut (Canada)", the authors, Jane Preston, Tim R. Claypool, William Rowluck and Brenda Green investigate the ways that a group of educators, Inuit and non-Inuit, understand traditional Inuit leadership and effective school leadership. The emphasis on an Inuit worldview that places great value on relationships and the concept of holism can be considered an alternative to other popular paradigms that positions educational leaders in managerial roles. The second article, by Roumiana Ilieva, Aojun Li and Wanjun Li, is entitled "Negotiating TESOL Discourses and EFL Teaching Contexts in China: Identities and Practices of International Graduates of a TESOL Program." In this article, the authors present the results of their study about the discourses circulating in a TESOL program in a Canadian university. Their study helps us to understand the complexities and conflicts involved with the shaping of teaching identities, in this case English as a Foreign Language teachers that occupy a "third space" between the local and the global, challenging conventional psychological models of identity as a fixed construct.

The next three articles shift our attention outside of Canada, and continue with this theme of alternatives. In our French article, "Using strength in numbers to support the development of reading fluency in sub-Saharan Africa", the authors Élisabeth Boily, Chantal Ouellet and Catherine Turcotte, demonstrate how the implementation of an alternative approach to reading instruction in Burkina Faso, called "assisted repeated reading", provided students with opportunities to improve their fluency development, a significant achievement in the resource-poor, large classrooms that constitute most schools in Burkina Faso. Using students as tutors allows for individual support to each student, thus effectively "drawing strength in numbers". The next article also focuses on the challenges faced by students from low-income backgrounds. In "Successful' Alternative Education: Still reproducing inequalities? The Case of the Community School Program in Egypt", authors Lucy El-Sherif and Sarfaroz Niyozov, explore the effects of the community school program, an alternative, empowering model of education, on students who had participated in this program. Their results are rather surprising in showing the unintended consequences associated with implementing alternative, progressive educational reforms. In our final article, "The centrality of participant voice in illuminating the gender regime in education research using a human capabilities analysis", the author Catherine Vanner examines the use of an alternative analytical framework for researching gender equality: the human capabilities approach. This approach has been used in a number of different educational studies to privilege participant voice and contribute to our understanding of the effects of the gender regime in schools as well as to provide alternative possibilities for creating a more empowered and gender just world.

Finally, we are pleased to have two book reviews by Comparative and International Education Society of Canada (CIESC) members in this last issue of the year. Christina Parker reviews Revisiting the Great White North? Reframing Whiteness, privilege, and identity in 
education, edited by Darren Lund and Paul Carr, members of the CIESC. And we end our December issue with a review of Dana Burde's Schools for conflict or for peace in Afghanistan by Stephen Bahry. Both of these books also contest normalizing discourses and provide us with alternative ways to guide our way forward as comparative and international educational practitioners and researchers.

We conclude our editorial with a telling quote from the French social theorist Michel Foucault. While he did not himself embrace the use of the word alternatives in his work, much of his writing does inform our thinking about the need to engage in critical analysis in order to challenge taken-for-granted assumptions about, in our case, normalized educational and research practices and approaches. Social change can only occur once we engage in this type of analysis, which we see in the articles in this issue, and which enables us to realize that there are alternative approaches to traditional pedagogies and school-based models, as well as different research approaches to consider. As Foucault (1988) writes:

Criticism is a matter of flushing out that thought and trying to change it; to show that things are not as self-evident as once believed, to see that what is accepted as self-evident will no longer be accepted as such...In these circumstances, criticism (and radical criticism) is absolutely indispensable for any transformation. A transformation that remains within the same mode of thought, a transformation that is only a way of adjusting the same thought more closely to the reality of things can merely be a superficial transformation. On the other hand, as soon as one can no longer think things as one formerly thought them, transformation becomes both very urgent, very difficult, and quite possible. (p. 155)

Until next time,

Marianne A. Larsen (English Editor)

Grazia Scoppio (French Editor)

\section{Éditorial: Volume 44 (numéro 2), 2015}

Nous sommes ravis de partager avec vous le dernier numéro du journal Éducation Comparée et Internationale. Comme dans les précédentes éditions, nous avons une autre série d'excellents articles traitant des thèmes et sujets variés d'éducation comparée et internationale, $\mathrm{y}$ compris une fois de plus, un article en français. En relisant les articles, un thème commun émerge - alternatives, ce qui est rafraichissant compte tenu de la standardisation et de l'homogénéisation des pratiques et approches éducatives en vigueur de nos jours.

Pour commencer, nous avons deux articles qui se concentrent sur les contextes éducatifs canadiens, quoique le second s'engage dans des discours éducatifs en Chine, de même qu'au Canada. Dans le premier article, «Explorer les concepts de leadership Inuit traditionnel et le leadership éducatif efficace, à Nunavut (Canada) », les auteurs, Jane Preston, Tim R. Claypool, William Rowluck et Brenda Green enquête sur les manières dont un groupe d'éducateurs, Inuit et non-Inuit, comprennent le leadership traditionnel des Inuits et le leadership scolaire efficace. L'emphase mis sur une vision mondiale Inuite qui accorde une grande valeur aux relations et au concept de l'holisme peut être considéré comme une alternative aux autres paradigmes populaires qui positionnent les leaders scolaires dans des rôles managériaux. Le deuxième article par 
Roumina Ilieva, Aojun Li et Wanjun Li est intitulé « Négocier les discours sur le TESOL et les contextes d'enseignement de l'EFL en Chine: l'identité et les pratiques des diplômés internationaux d'un programme de TESOL ». Dans cet article, les auteurs présentent les résultats de leur étude sur les discours circulant dans un programme de TESOL dans une université canadienne. Leur étude nous aide à comprendre les complexités et les conflits inhérents à la formation des identités d'enseignant; dans ce cas précis, des enseignants d'anglais comme langue étrangère qui occupent un « troisième espace » entre le local et le global, mettant à défi les modèles psychologiques conventionnels d'identité en tant que construction fixe.

Les trois prochains articles déplace notre attention en dehors du Canada, et continue avec ce thème d'alternatives. Dans notre article en français, «Utiliser une force du nombre pour supporter le développement de la maîtrise de la lecture en Afrique Sub-Saharienne », les auteurs Élisabeth Boily, Chantal Ouellet et Catherine Turcotte démontrent comment, au Burkina Faso, l'implémentation d'une approche alternative d'instruction à la lecture, appelée « lecture assistée et répétée », a fourni aux élèves des opportunités d'améliorer le développement de leur fluidité, ce qui représente un accomplissement significatif dans les larges classes à pauvres ressources que constituent la plupart des écoles du Burkina Faso. Utiliser des élèves en tant que tuteurs permet un support individuel à chaque élève, permettant efficacement de «tirer de la force dans le nombre. » Le prochain article porte également l'accent sur les défis auxquels font face les étudiants issus de milieux à faible revenu. Dans «L'éducation alternative 'à succès' : Reproduisant encore les inégalités ? Le cas d'un programme scolaire communautaire en Égypte », les auteurs Lucy El-Sherif et Sarfaroz Noyozov explorent les effets du programme d'école communautaire, un modèle alternatif et autonomisant d'éducation, sur les élèves qui avaient participé à ce programme. Leurs résultats étaient plutôt surprenants en démontrant les conséquences imprévues associées à l'implémentation de réformes éducatives alternatives et progressives. Dans notre dernier article, «La centralité de la voix du participant pour éclairer le régime de genre dans la recherche en éducation en utilisant une analyse des capacités humaines », l'auteur Catherine Vanner examine l'utilisation d'un cadre analytique alternatif pour la recherche sur l'égalité des sexes : l'approche des capacités humaines. Cette approche a été utilisée dans un certain nombre d'études éducatives différentes visant à favoriser la voix du participant et à contribuer à notre compréhension des effets du régime de genre dans les écoles, de même qu'à offrir des possibilités alternatives pour créer un monde davantage autonomisant et plus juste entre les sexes.

Finalement, dans ce dernier numéro pour l'année, nous sommes heureux d'avoir deux critiques littéraires écrites par des membres de la Société Canadienne d'Éducation Comparée et Internationale (SCÉCI). Christina Parker critique Révisiter le Grand Nord Blanc? Recadrer la blancheur, le privilège et l'identité en éducation, édité par Darren Lund et Paul Carr, membres de la SCÉCI. Et nous terminons notre numéro de décembre avec une critique littéraire de Dana Burde sur Les écoles pour le conflit ou pour la paix en Afghanistan par Stephen Bahry. Ces deux livres contestent également les discours qui normalisent et nous offrent des façons alternatives en vue de guider notre chemin vers l'avant en tant que praticiens et chercheurs en éducation comparée et internationale.

Nous concluons notre éditorial avec une citation révélatrice du théoricien social français Michel Foucault. Quoiqu'il n'ait pas lui-même embrassé l'utilisation du terme 'alternatives' dans son travail, une bonne partie de ses écrits pousse notre réflexion sur la nécessité de se livrer à une analyse critique afin de défier les assomptions prises pour acquises concernant, dans notre cas, les pratiques et approches d'éducation normalisée et de recherche. Le changement social ne peut se 
produire que si nous nous engageons dans ce genre d'analyse que nous trouvons dans les articles de ce numéro, et qui nous permet de réaliser qu'il existe des approches alternatives aux pédagogies traditionnelles et aux modèles basés à l'école, de même que des approches de recherche différentes à considérer. Comme Foucault (1988) l'écrit:

La critique est une manière de débusquer cette pensée et d'essayer de la changer; afin de montrer que les choses ne sont pas aussi évidentes qu'on l'a une fois cru, de voir que ce qui est accepté comme allant de soi ne sera plus accepté comme tel... Dans ces circonstances, la critique (et la critique radicale) est absolument indispensable pour toute transformation. Une transformation qui demeure dans le même mode de pensée, une transformation qui représente seulement un moyen d'ajuster la même pensée plus proche de la réalité des choses, peut difficilement être une transformation superficielle. D'un autre côté, aussitôt que l'on ne pense plus aux choses de la même manière qu'on le pensait auparavant, la transformation devient à la fois très urgente, très difficile, et tout à fait possible (p. 155).

À la prochaine,

Marianne A. Larsen (rédactrice en chef, version anglaise)

Grazia Scoppio (rédactrice en chef, version française) 